\title{
Innovative Research in Visual Communication Design and Its Enlightenment on Teaching
}

\author{
Shanshan Gong
}

Art college of xi'an university, 710065

Keyword: Visual communication design; Thinking mode; Design concept; Innovation; Teaching

\begin{abstract}
The design of visual communication is closely related to thinking mode and design concept. In some ways, the three are interlinked. Therefore, it is necessary to attach importance to the innovative research on thinking mode and the design concept of visual communication so as to effectively improve the design quality. This paper discusses and analyzes the innovation of visual thinking and design concept and summarizes the enlightenment of innovative teaching on talents.
\end{abstract}

\section{Introduction to Visual Communication Art Design}

Art design related to visual communication refers to that visual designers make reasonable collocation of the design elements according to specific rules and standards and then analyze and combine them so as to make the final effect express the intentions of the designers, i.e., texts, graphics, audios and other visual elements are applied comprehensively so as to produce visual impact on people finally and to make sure that audiences can understand the contents of design, thus meeting people's visual demands. In addition, it can also create ideal value for those who use visual design and demonstrate the visual effect.

For the current art design in China, the artistic color is more obvious. Great development has been made in terms of both aesthetics and artistic value. However, there is still no major change in essence, but its purpose is still to deliver the visual information and the improvement of the design is also to make the communication more reliable and effective. However, with the development of this communication design, its scope of application is bound to continue to expand and promote. Especially, in terms of business, art design of visual communication can well serve for business so as to make audiences more understand and recognize the company, and this also well promotes the corporate image and products. It can be said that the development of visual design in business is its practical promotion. At the artistic level, significant progress and optimization has also been made in this design. The artistic design of visual communication is constantly updating and optimizing its artistry while promoting the development of the entire field of visual communication so as to move it forward in practical fields.

\section{Innovation of Visual Communication Mode of Thinking}

Direction of Thinking Mode Innovation. Relationship between Coordination Proportion and Scale In order to innovate the thinking mode of design, the first thing is to coordinate the relationship between scale and proportion. Among people's five sense organs, organs related to visual sense has the most obvious and direct impact, mainly because visual is people's first feeling in identifying things. For many times, we use our eyes to recognize and know the world. Therefore, in promotion, many enterprises will advertise their own products with ads. To innovate thinking mode, it is necessary to insist on the coordination of proportion and scale.

In order to coordinate the scale and proportion, it is necessary to be appropriate, i.e., we need to accurately grasp the size of the picture or video. If the design is too small, it cannot attract the audiences' attention, and if the design is too large, they cannot make the audiences accept all the information. Therefore, it is necessary to grasp the degree of and relationship between proportion and scale. It cannot be too large or too small, and it is better to choose the golden proportion, which must be followed particularly in designing posters. In addition, to coordinate proportion and scale can make people feel beautiful and enhance the proportion effect so as to make enterprises gain 
more economic and social benefits. In the innovation of thinking mode, it is necessary to determine the direction and optimize the coordination of scale and size so as to enhance the visual effect.

Coordinate the Relationship between the Part and the Whole

In the construction of design graphics or videos, designers need to reasonably coordinate the local part and the entire part. However, it is difficult, because we always choose the highlighted points in design, and sometimes, in order to embody the highlights, it is necessary to make special design and achieve particular effect. Therefore, sometimes, the local design will go contrary to the entire design. Although it can still bring visual impact to audiences, it cannot leave a good impression on audiences. Therefore, in innovative design, it is necessary to coordinate the local and entire relationship so as to make the design give people a harmonious feeling.

Insist on Being Visual-Centered

The most important part of visual communication and design is "visual", so in the innovation of visual thinking, it is necessary to take "visual" as the center of the design and the entire design should focus on this point. Meanwhile, there is a need to take the visual sense as people's sensory channel to observe the world. It can quickly concentrate people's interest and then make people identify the objects within a short time. Therefore, designers need to realize the importance of visual in design so as to make audiences become more interested in enjoying the design works so as to achieve the design effect, expand the influence of design and bring more benefits for customers.

Pay Attention to the Relationship between Symmetry and Equilibrium

Symmetrical shapes make people seem more natural and stable, and make the entire picture have a uniform and neat beauty, which is in line with people's habits of looking at things. We can see symmetrical shapes everywhere in our lives, such as the shape of cars and animal bodies, etc. Therefore, we should make full use of the common points of people's aesthetics, fully utilize the image thinking and a variety of vivid materials and then reasonably combine them so as to generate new images or convert them into other images. However, in information processing, it is not a series of processing, but the parallel processing mode, which is plane or three-dimensional so as to make the thinking subject quickly grasp the issue from an overall perspective.

Different from symmetry, balance is an asymmetrical representative of balance. Symmetry is absolute, while balance gives people a sense of coordination between vision and psychology. When people look at any object, they will seek a balanced and stable state. Balance is actually more of people's psychological experience and results from the stable state with the same form but different quantities.

\section{Emphasize on the Relationship between Contrast and Unity}

Different contrasts have their own characteristics both $n$ value and in effect, which is also the most commonly used means of visual communication. For example, through the contrast of the bright and dark hue and the color saturation, it can fully reflect the visual effects. And unity is to arrange the contents in an orderly manner and it is the overall grasp of beauty. Art works are the result of the author's orderly unification of the contents according to his inner world. Therefore, importance must be attach to the relativity contained in unity in the demonstration of visual design. It is necessary to grasp the relationship between unity and contrast and fully reflect the aesthetic flavor contained in works.

\section{How to Make Innovations in Thinking Mode}

Emphasis on Reverse Thinking. Innovation means to start from a reasonable standpoint but appears to be unique, and it is to do something unconventional or unorthodox in succession. Reverse thinking, on the other hand, seems to be a way of thinking about a determined subject from the other end. This model will lead people in the opposite direction, make designers break away from the more conventional ideas and make creative combination or design of the existing design elements again.

Therefore, attention must be paid to the training of reverse thinking mode in visual communication related work. Designers need to "go the opposite way" in design and pay attention to the beauty contained in the surrounding environment in life at any time. Perhaps a casual 
observation may constantly produce inspiration. Without unique feature, the design's visual effect may become dull. In addition, reverse thinking is not simply to think reversely, but it must have a reliable basis and there must be a chance to realize it while thinking of what cannot be thought of by others. This is the reverse thinking in the true sense. It breaks people's usual thinking rules. When designers think and design in this reverse form, they often achieve amazing results.

Emphasize on Divergent Thinking. In order to innovate in visual design, the designers should not think too simple, but should divert their focus to the entire situation, think and design works with a divergent thinking. Only in this way can the visual works become more in line with the interest and aesthetic taste of the public. If the designers only focus on one point, the design works may not become comprehensive. Therefore, it is necessary to make sure that the designers must have comprehensive thinking in design without simply thinking from one point. The design must be thought of comprehensively and start from various angles to think of and construct the design. Various design points should be used and connected to form a plane so as to make the design more perfect and comprehensive.

Emphasize on Associative Thinking. The power of both imagination and association is needed in design. Imagination can provide designers with more inspiration and help them design unique work, and this is also the most basic thinking for designers. Association is greatly different from imagination. Imagination seems to be more unrestrained and does not have any rules to follow. It only relies on people's own imagination. Association starts from reality and is based on the real things. In association, the rational thinking is used to find the common characteristics of all things, which are used to fully associate things. For example, upon seeing chocolate, many people will associate it with the Valentine's Day, and this is association. Therefore, association must have a basis. In association, based on people's thinking, people have rational thinking. Therefore, designers must pay attention to the application of associative thinking in innovative design so as to make the design works more in line with the social demands and become more "rooted".

\section{Innovation in Visual Communication Design Concept}

Innovate the Design Concept. It is not enough for designers to rely solely on the innovation of thinking mode, and it is necessary to innovate and optimize the design concept, which must be combined with the real demands of the external environment because visual works will finally be embodied in practical application, and the value of such practical application must meet people's various visual demands. Therefore, while innovating and optimizing the design concept, designers must start from the demands for visual design in all industries and update the design concepts according to demands in this industry so that the designers can closely follow the track of the times and promote the visual works more reflect the timeliness and become in line with the development trend of the times.

Innovate Visual Elements. In addition to innovating concepts, it is also necessary to innovate and optimize the specific contents. And at the content level, innovation is mainly for visual elements. Particularly, visual language should be constantly innovated. The real innovation and optimization of visual elements involve many contents, such as colors, various graphics and animation, etc. All these require innovation in accordance with the development of the times so as to make the design works more reflect features of the times and become able to attract audiences at every period of time. Meanwhile, designers should avoid use obsolete or unappealing elements, but they should use the new features or new culture demonstrated by the time so as to achieve the most ideal visual effect.

Innovate the Way to Express Visual Communication. If expression of different forms is used in visual communication design, it will finally produce great difference in the final visual effect, which is also a link that should be particularly studied by designers at current stage. In innovative research on performance patterns, attention must be paid to the adaptation to the outside world. It is necessary to make corresponding innovation according to the various demands for products and the different audiences so as to make the works more creative. Designers need to pay attention to the requirements at various aspects while innovating the expression ways in reality. They should not 
only pay attention to the various external demands, but also attach more importance to the innovation of expression ways and constant enrich various ways of expression so as to improve the innovation value of works, which is also the key to the innovation of visual communication.

\section{Carry out Innovative Teaching to Talents}

\section{Grasp the Features of the Times}

The Teaching of Visual Communication Related Design Must be Based on the Demands for Different Talents. It is necessary to grasp the time and carry out targeted teaching. The teaching of visual design at current stage still needs to be based on the traditional system, but targeted improvement measures should also be taken according to features of the times. For example, while explaining the advertising planning, teachers should not only let students learn the advertising planning and the design of promotional texts and print advertisements but also add suitable advertisements in the form of videos and network so as to make the teaching contents more enriched. In addition, students can also learn 3DMAX and other software that are more in line with the trend of the times so as to better grasp the features of the times.

Improve the Existing Curriculum. In the teaching of visual communication design at the current stage, many contents are already too obsolete. Therefore, teachers need to properly improve the teaching contents of visual design. For example, innovation and design course related to UI can be set at school. At present, there are more and more needs for visual designers in the Internet industry, software industry and other industries. To carry out this course can effectively meet the demands for talents so as to make the teaching more responsive to the times.

\section{Conclusion}

This paper mainly discusses and analyzes the thinking modes and design concepts related to visual communication design in-depth and proposes corresponding measures for improvement. This not only provides novel design thinking and concept but also proposes new teaching enlightenment for teaching related to visual communication. This is the innovation of not only the visual communication industry, but also the promotion and media industry with important guiding significance for improving the corporate economic and social benefits and promoting social development.

\section{References}

[1] Wang Yuzhao. Explore the Implementation of Innovative Design Concepts in Visual Communication Art Design [J/OL]. Art Technology:(2017-10-30).

[2] Liu Jiaying. Analysis of Innovative Design Concept of Visual Communication Art Design [J]. Popular Literature and Art, 2017, (11):108.

[3] Tong Fei, Wu Qiong. Research on the Current Situation and Countermeasures of Visual Communication Design Talents Training Based on Collaborative Innovation [J]. Technology Outlook,2016,26(16):289.

[4] Wang Le. Analysis of the Concrete Implementation of Innovative Design Concept in Visual Communication Art Design [J]. Packaging World, 2015, (06):30-31.

[5] Ding Lei. Innovative Research on Integrated Experimental Course of Visual Communication Design Specialty in Digital Media Environment [J]. Art and Design (Theory),2012,2(10):166-168.

[6] Liu Lifu. Reflections on the Cultivation of Innovative Thinking in Visual Communication Design in the New Era [J]. Art and Design (Theory), 2010, 2(04):146-148. 
[7] Qi Yue Chun. Aesthetic Characteristics of Characters in Visual Communication Design [J]. Journal of Xiamen University (Philosophy and Social Science Edition), 2008(12).

[8] Wang Xuebin, Zhao Dongsheng. Influence of Carving Art on Visual Communication Design and Its Enlightenment [J]. Hunan Normal University: Journal of Design, 2009(1).

[9] Zhu Yongming. Reflections on the Relationship between the Development of Visual Communication Design and the Visual Cultural and Creative Industries [J]. Press,2009(5).

[10]Li Xiaobing. Traditional Chinese Culture and Modern Network Visual Communication Design [J]. Qingdao University: Journal of Design,2009(13).

[11] Qiang Xiaoning. The Use of Traditional Graphics in Modern Visual Communication Design [J]. Movie Review, 2009(10).

[12]Men Delai. Modern Graphic Design Idea and Performance [M]. Xi'an: Xi'an Jiaotong University Press, 2002. 